\title{
The evolution of a thermocline effected by a turbulent stream
}

\author{
O. A. Druzhinin, V. I. Kazakov, P. A. Matusov and L. A. Ostrovsky \\ Institute of Applied Physics of the Russian Academy of Sciences, 46, Uljanov St., Nizhny Novgorod 603600, Russia
}

Received 31 August 1994 - Accepted 21 December 1994 - Communicated by A. Babiano

\begin{abstract}
The process of thermocline evolution under the action of turbulent stream in the upper layer is investigated in laboratory experiment in thermally stratified tank, the initial temperature profile with pronounced thermocline being similar to that observed in tropic seas. The mean velocity and turbulent energy spatial distributions have been shaped to model the hydrological conditions in strong oceanic currents or wind-induced drag currents.

The experiment demonstrates the gradual deepening and transformation of thermocline in a case where no global instability took place (i.e., with Richardson numbers always exceeding $0.3-0.4$ ). The process of thermocline evolution resulted also in recurring "bursts" of microstructure.

A numerical experiment based on equations of semi-empirical theory of turbulence showes quantitative agreement with experimental data. Moreover, simple analytical solutions and numerical results show that a layer with marginal stability is formed with Richardson numbers being very close to the stability threshold, so that quite small disturbances in thermocline can result in appearance of internal waves and bursts of turbulence.
\end{abstract}

\section{Introduction}

The process of mixing in the thermocline under the action of turbulence is of interest for oceanography, meteorology and other fields of geophysics. Sources of turbulence in geophysical flows are typically related to the action of wind stress on the sea surface, breaking of internal waves, etc.

Processes of this type were investigated theoretically by Zilitinkevitch et al. (1988) and Zilitinkevitch and Mironov (1989), in laboratory experiment with wind-induced mixing by Kreiman and Bogdanov (1990), an interesting observation in sea was made by Artale et al. (1988) showing the intermitting character of thermocline transformation. Kennedy and Shapiro (1980) have reported an investigation of the clear air turbulence where they focused their attention on the microstructure. Some laboratory experiments with turbulence in density- stratified fluids are known (see, for example, the review by Britter (1988) and more recent results obtained by Perera et al. (1994)).

However, most studies mentioned above concern the situation when a source of turbulence, which brings about mixing, is distributed uniformly at the surface of the fluid (as it is, e.g., for a wind flux over sea surface, or for an oscillating grid (Kato and Phillips, 1969; Xuequan and Hopfinger, 1986). Moreover, the experiments are performed mostly in some model situations like two-layer fluid or continuous stratification with uniform vertical profile of buoyancy frequency.

On the other hand, there are situations when mixing is caused by a stream near the water surface, when turbulence and velocity fields have a jet-like structure. This is the case e.g. in the vicinity of a river mouth in the ocean, or near strong ocean currents like the Gulf Stream, or the wind drag during strong storms. The processes of the intrusion of a heated plane jet into an ambient cool fluid was investigated by Antonia et al. (1986). In particular, the dynamics of organized vortex structures and their influence on the temperature front were studied. In a more recent work (Sutherland and Peltier, 1994) the evolution of a symmetric jet in a density stratified fluid was simulated numerically. Results obtained show that due to the processes of vortex pairing internal gravity waves can be radiated efficiently for certain types of the density profiles. Corresponding laboratory experiments with explicit modeling of the phenomenon are of particular interest.

In this paper we present some results of study of the thermocline transformation under the action of turbulent stream near the water surface, which was performed in the thermally stratified water in a tank provided with a turbulent flow producing tool. Performing the laboratory experiment and numerical calculations we assumed that the process of rotation are unimportant, so that the resuts discussed are applicable for the corresponding geophysical processes on the scales small enough to neglect the Coriolis force. The experiments have been conducted in the conditions similar to those observed in tropic seas and atmosphere, with vertical distribution of temperature with pronounced thermocline. 


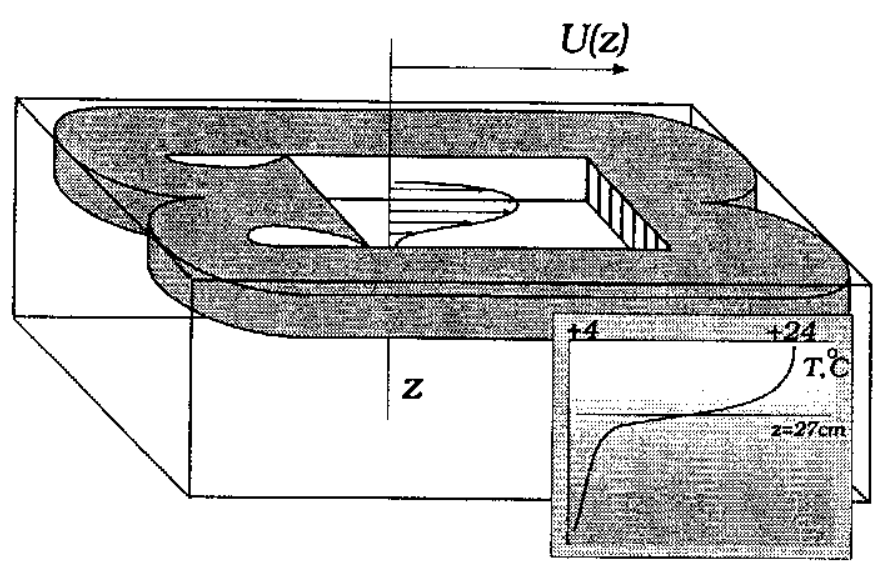

Fig. 1. Schematic view of experimental setup.

\section{Experimental setup, measuring technique and model coefficients}

The sketch of the experimental setup in presented in Fig. 1 (see the details of construction in Arabadzhi et al. (1984). The tank was $5 \mathrm{~m}$ long, $1.6 \mathrm{~m}$ wide and $1.2 \mathrm{~m}$ deep. Special heatexchanging equipment produced the thermal stratification so as the temperature near the water surface was $22-24^{\circ} \mathrm{C}$, the bottom temperature was $4-8^{\circ} \mathrm{C}$. Thus, the temperature jump was very close to that in the real sea. The sharp thermocline laid at $27 \mathrm{~cm}$ under the water surface. Typical values of the buoyancy frequency

$N=\left(-\frac{g}{\rho} \frac{\partial \rho}{\partial z}\right)^{1 / 2}$

were about $0.31-0.39 \mathrm{rad} / \mathrm{s}$ (buoyancy periods from 16 to $20 \mathrm{~s}$ ) in the beginning of experiments. A "jet inductor" was introduced in the upper layer of water. The stream was $20 \mathrm{~cm}$ thick at the outlet of the jet inductor; the flow velocity mean laid in the range from 1.5 to $8.5 \mathrm{~cm} / \mathrm{s}$, with the turbulent pulsations about $30-50 \%$ of the mean value.

It should be mentioned that since the temperatures near the surface and below the thermocline are close to those in nature conditions, and characteristic depth of sharp thermocline $(27 \mathrm{~cm})$ is about 100 times less than that in the sea (buoyancy period scaling factor about 10 ), the corresponding minimal Brunt-Vaisala periods in the seasonal thermocline in the sea should be about 3 minutes, as it is actually observed.

Another question is about the model coefficient for the mean velocity. Indeed, if we perform the experiments with approximately the same Richardson and Froude numbers

$$
\begin{aligned}
R i & =\frac{N^{2}}{(\partial U / \partial z)^{2}} \sim \frac{N^{2} L^{2}}{U^{2}}=\left(\frac{n l}{u}\right)^{2} \\
F r & =\frac{U}{N L}=\frac{u}{n l}
\end{aligned}
$$

where capital letters denote the characteristic parameters in the sea conditions and small letters-those in the experiment, we get the scaling factor for mean velocity about 10 , thus, the range of mean velocities in laboratory experiment from 2 to $10 \mathrm{~cm} / \mathrm{s}$ corresponds to that from 0.4 to 2 knots in the sea, which is typical for ocean situations.

In the experiments the temperature and velocity profiles have been measured. Temperature profiling was performed with $1 \mathrm{~mm}$ spatial and $0.1 \mathrm{~s}$ temporal resolution; the scanning was periodical with $1.5 \mathrm{~min}$ delay (thus, internal waves have not been measured by the scanning probe). The velocity profiles were measured at $15 \mathrm{~cm}$ from the stream outlet (diffusor), $15 \mathrm{~cm}$ from the stream inlet (confusor) and in the middle of the tank ( $100 \mathrm{~cm}$ from the diffusor) with $1 \mathrm{~cm}$ spatial resolution and usually three times during the experiment-at the very beginning, 15 minutes and 1.5 hours after the beginning. During the experiments the continuous temperature measurements at several fixed points were performed to study the internal waves. The temperature profiles were digitized and processed numerically to obtain information about the density and buoyancy frequency spatial distribution.

This study considers mainly the transient process of thermocline evolution in rapidly changing external conditions, and all the experiments discussed here have been performed without heat forcing during the measurements. However, some measurements have been made with continuous maintaining of temperature at the surface and bottom demonstrating no difference at the initial stage (about 25-30 min after the beginning) and qualitatively similar behavior later.

\section{Observations}

The temperature, $N^{2}$ (squared buoyancy frequency) and mean velocity profiles measured in the middle of the tank in the very beginning of the experiment are illustrated by Fig. 2 . It can be seen that the maximum of Brunt-Vaisala frequency lays at the depth about $30 \mathrm{~cm}$ with a buoyancy period about $20 \mathrm{~s}$, while the mean velocity exhibits fast decreasing at 20$25 \mathrm{~cm}$. This results in the appearance of the unstable layer with $R i \sim 0.1$, that should lead to instability and fast mixing near the thermocline. The mean flow velocity profiles have been measured at three locations $(15 \mathrm{~cm}, 1 \mathrm{~m}$ and $2.25 \mathrm{~m}$ apart from the stream outlet along the central line of the tank). It should be noted that the mean velocity profile varies with the distance from the diffusor (see solid curves in Fig. 3). The stream appears to be slightly diverging with an angle about $9-13^{\circ}$ and not remarkably changing during the experiment. At the right hand plot a weak flow below $40 \mathrm{~cm}$ depth is observed; possibly, this is a drag current below the thermocline with opposite direction (unfortunately, we were not able to measure the the velocity vector). The effect of stratification on a velocity profile is illustrated by dashed curves in Fig. 3. The velocity profile in the experiment without stratification is much smoother and wider than that in a case of stratified water. In stratified fluid the velocity profile adjusts itself so as the region with intensive shear lays close to that with maximum density gradient. This plays the main role in the evolution process as it will be shown below. 


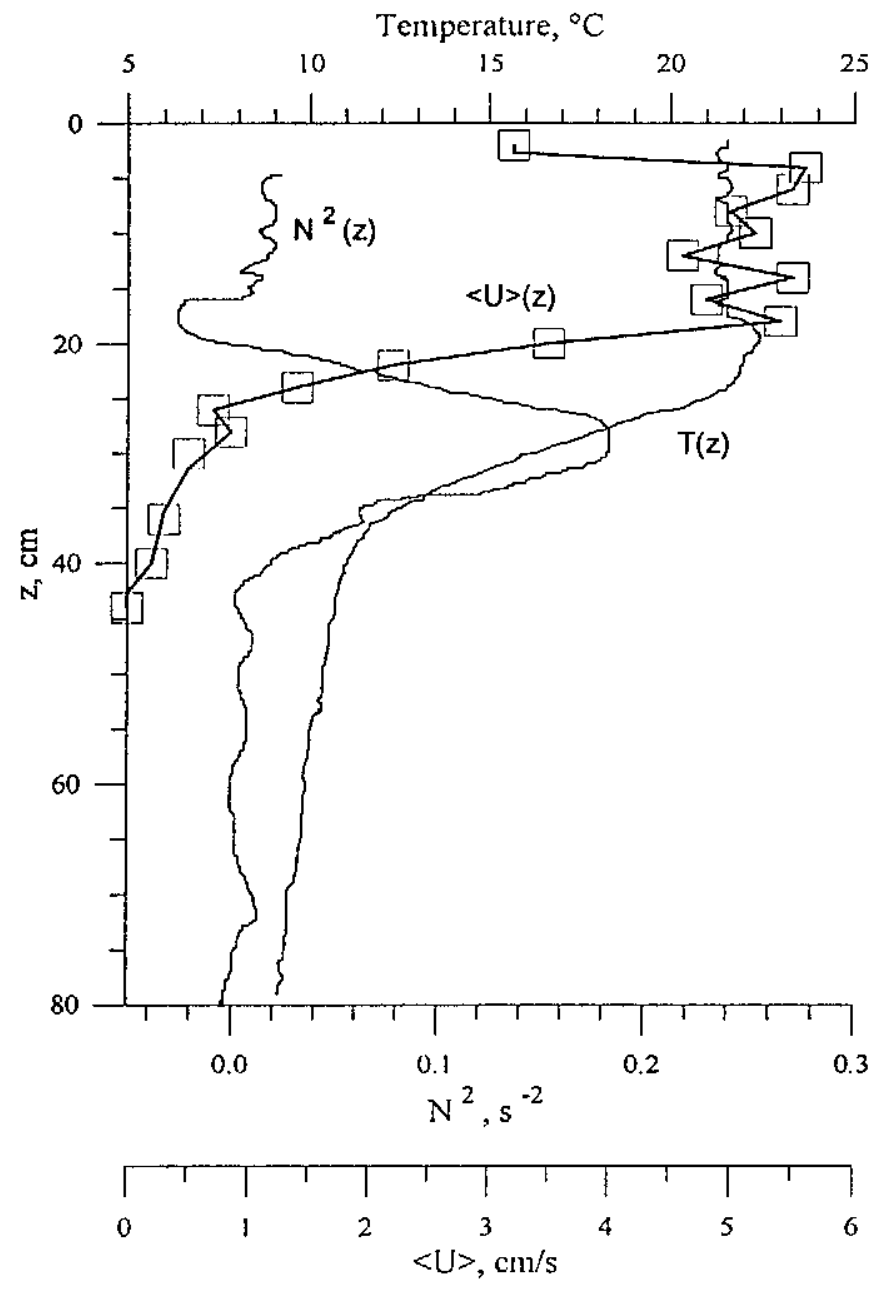

Fig. 2. Vertical profiles of temperature, Brunt-Vaisala frequency and mean flow velocity (squares and thick curve).

The pulsation velocity component has also been measured in the experiment. It was found that in the beginning of the experiments vertical distribution of turbulent energy has been similar to that of mean velocity, with characteristic turbulence level $U_{r m s} /\langle U\rangle \sim 30-50 \%$. We recorded several realizations of turbulent pulsations at several depths and processed these realizations using an FFT algorithm. A bright peak in power spectra of pulsations is observed at $6.8 \mathrm{~Hz}$ at $20 \mathrm{~cm}$ below the water surface. Possibly, this may be interpreted as existence of pulsations with a certain lengthscale about $0.7 \mathrm{~cm}$. This hypothesis is also implied by presence of a large vortexbreaking grid inside a jet inductor with a $1 \mathrm{~cm}$ mesh size.

The turbulent stream causes essential changes in a thermocline (see Fig. 4)-the upper layer temperature becomes almost uniform, the jump layer sharpens the density inversion layers appeares. The intensive internal waves are excited immediately after the switching on of the inductor (probably, this is due to a pressure jump near the diffusor), but they decay gradually and after 5-10 minutes after the beginning of experiments the waves are absent.
Some time later (about 20-30 minutes) a remarkable fine structure of the temperature spatial distribution appeared. The mixing process due to the turbulent stream caused also a gradual deepening of the layer with the buoyancy frequency maximum. Figure 5 represents the temperature spatial redistribution during the experiment with flow velocity mean $5 \mathrm{~cm} / \mathrm{s}$. Here each thin curve represents an isotherm. The place where these curves are very close to each other is that with the density gradient maximum. One can see in the plot that the temperature jump layer is gradually deepening and smoothening. A regions with families of closed curves mean the appearance of the density inversions.

While discussing the observations, we defined a thermocline location in a simple manner:

$z^{*}=\int_{z=0}^{z=H} z N^{2}(z) d z / \int_{z=0}^{z=H} N^{2}(z) d z$,

where the integrals were calculated over the depth interval from zero to $H=80 \mathrm{~cm}$ (the maximal depth available for the temperature scanner). We examined the temporal variation of this value with time in a set of experiments with flow velocities ranging from 2 up to $7 \mathrm{~cm} / \mathrm{s}$. The results are shown in Fig. 6. The families of experimental points have been approximated by the square polynom curves. One can see that in all cases the thermocline has been deepening with the characteristic rate depending on the mean flow velocity. The initial thermocline deepening rate as a function of the flow velocity average is shown in Fig. 7. The solid line is a linear empirical law introduced by Kato and Phillips (1969) for turbulent mixing in thermocline.

The temporal behavior of the buoyancy frequency $\mathrm{N}$ over the thermocline in the experiment with mean velocity $5 \mathrm{~cm} / \mathrm{s}$ is shown in Fig. 8 (empty squares). In experiments it was found that this value reached a maximum and then gradually decreased. Filled triangles and thick curve represent this dependence obtained in numerical experiment that will be discussed below.

Spatial distributions of mean velocity may be used together with temperature profiles to calculate the Richardson numbers $(R i)$ reached in these experiments. The calculations yield the values of $R i$ about 10-100 far from thermocline, and characteristic $R i$ numbers about 1 in thin layers the vicinity of thermocline. Together with the absence of significant internal waves that means that global Kelvin-Helmholtz instability does not take place in the system, and the thermocline evolution is caused mainly by turbulent mixing. However, these thin layers near thermocline with strong velocity shear and weak stratification can be an additional source of turbulence, which remains to be investigated.

The process of thermocline evolution is often accompanied by the fine structure formation (see Fig. 9). The plot represents 7 sequent temperature profiles measured with 1.5 minute interval in experiment with $7 \mathrm{~cm} / \mathrm{s}$ mean velocity. It is seen that almost immediately after the switching on of the jet inductor a density inverse layer is formed; then this density 


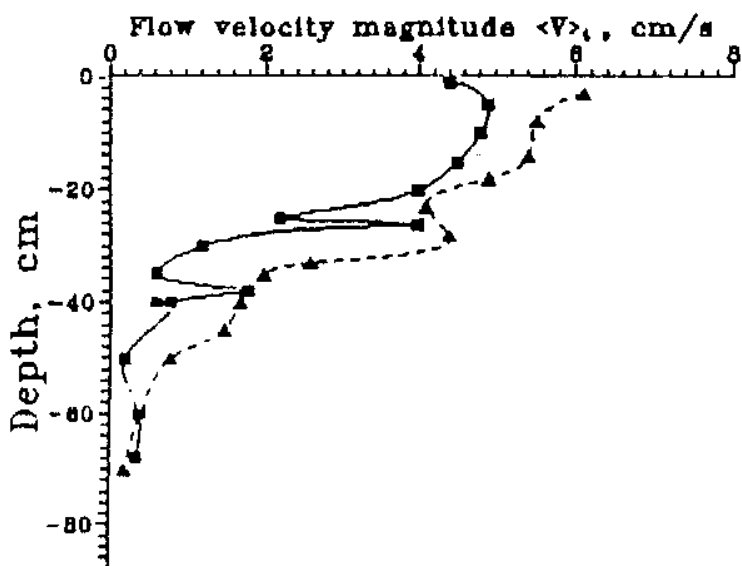

$226 \mathrm{~cm}$ from the outut.
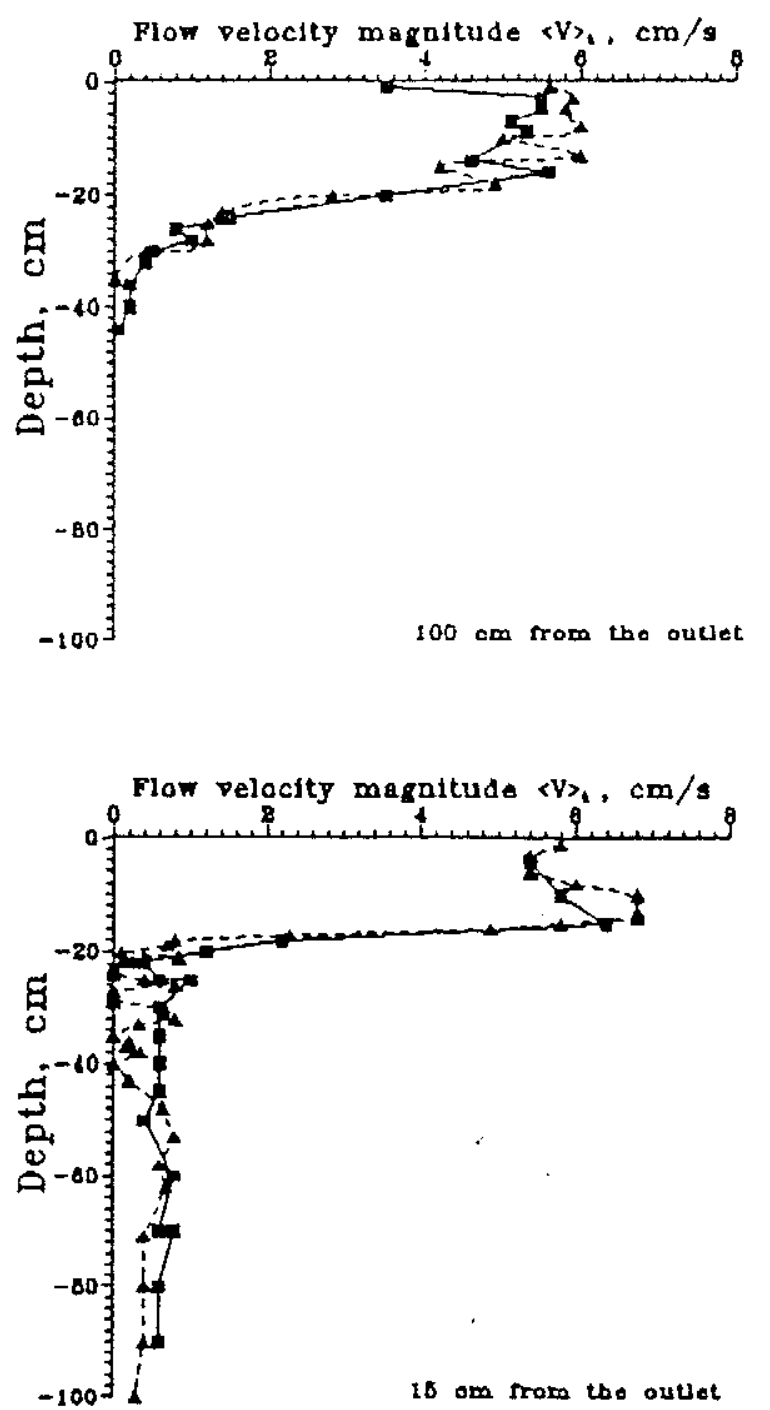

Fig. 3. Vertical profiles of mean velocity measured at $15 \mathrm{~cm}$ from the outlet of inductor, in the middle of working area (100 cm from the outlet) and $15 \mathrm{~cm}$ from the stream inlet $(225 \mathrm{~cm}$ from the outlet). Squares and solid curves represent the data obtained 15 minutes after the beginning of the experiments, triangles and dashed curves- 1.5 hours after the beginning. inversion breaks resulting in appearance of remarkable fine structure. This process is observed to be recurring, thus, exhibiting dynamical origin. Such a behavior of stratified fluid in presence of shear flow in a quite narrow range of global Richardson numbers from several tenths to several unities has recently been communicated by Gibson (1994). It is evident that knowledge of dynamical properties of this phenomenon is of considerable physical interest and may yield the tool for diagnostics of "large events" in thermocline. An attempt of statistical analysis of these data has been made by Kazakov et al. (1994).

\section{Theoretical model and numerical results}

In order to describe analytically the processes observed we applied well known approach based on the $k-l$ closure procedure (Rodi, 1980). As it follows from the experimental results and the whole geometry of the model, characteristic variations over horizontal coordinates are much smaller than those over vertical coordinate $z$. Thus, it is appropriate to consider a one-dimensional model with velocity, density and temperature field depending only on $z$, i.e. $V=\{U(z), 0,0\}$ and $\rho(z)$. Corresponding equations describing the dynamics of turbulence intensity, average velocity and density fields and density fluctuations take the form (Rodi, 1980)

$$
\begin{aligned}
\frac{\partial b}{\partial t}= & \frac{\partial}{\partial z} l \sqrt{b} \frac{\partial b}{\partial z}+l \sqrt{b}\left(\frac{\partial U}{\partial z}\right)^{2} \\
& -\kappa l \sqrt{b} N^{2}-\frac{\gamma b^{3 / 2}}{l}, \\
\frac{\partial U}{\partial t}= & \frac{\partial}{\partial z} l \sqrt{b} \frac{\partial U}{\partial z}, \\
\frac{\partial \rho}{\partial t}= & \frac{\partial}{\partial z} \kappa l \sqrt{b} \frac{\partial \rho}{\partial z}, \\
\frac{\partial P}{\partial t}= & \kappa l \sqrt{b} N^{2}-\frac{\gamma b^{1 / 2} P}{l}+\frac{\partial}{\partial z}\left(\kappa l \sqrt{b} \frac{\partial P}{\partial z}\right),
\end{aligned}
$$

where $b=\left\langle U^{2} / 2\right\rangle$ is turbulent kinetic energy (i.e., squared velocity averaged over an ensemble of realizations), parameter $\kappa$ is the ratio of temperature fluctuations scale and velocity fluctuations scale, $\mathrm{N}$ corresponds to Brunt-Vaisala frequency, empirical coefficient $\gamma=0.05$ and $P$ stands for an effective "potential" energy related to density dispersion function given by $P=g^{2}\left\langle\rho^{2}\right\rangle / 2 \rho^{2} N^{2}$ (Ostrovsky and Troitskaya, 1987). Since the flow may be regarded as a free turbulent flow, it is appropriate to assume that characteristic scale of velocity fluctuations $l$ remains constant (Rodi, 1980). Taking into account experimental observations, we choose $l=1 \mathrm{~cm}$ and ratio $\kappa$ equal to 1 . Note, that since the experiment was performed under temperatures in the interval $4-20^{\circ} \mathrm{C}$, the relation between fluid density and temperature is nonlinear. With sufficient accuracy it can be described by the expression

$T(z, t) \equiv 10^{3}\left(\frac{\rho(z, t)}{7.92 \rho_{0}}\right)^{1 / 3}$ 
Temperature, $C$

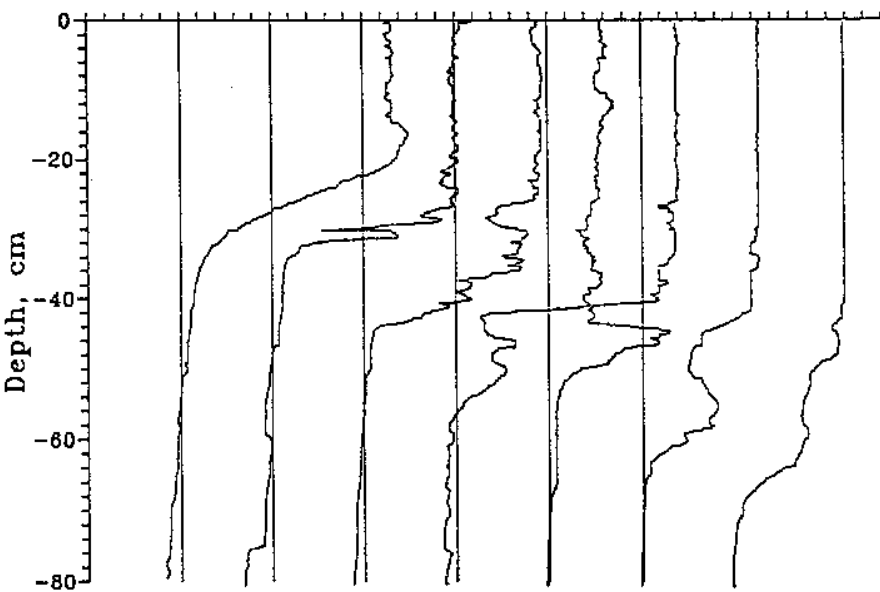

Flg. 4. Temperature profiles obtained in the experiment with $5 \mathrm{~cm} / \mathrm{s}$ mean flow velocity. The interval between neighboring profiles is 15 minutes.

From experimental data described above it follows that flow in the region close to the surface corresponding to the turbulent stream remains practically unchanged and is defined by the conditions at the outlet of the stream. Therefore, in region $z_{0}<z<0$ mean velocity and turbulence profiles can be regarded as stationary maintained by the stream and given by

$U=U_{s}(z), \quad b=b_{s}(z), \quad$ for $z_{0}<z<0$,

where functions $U_{s}(z)$ and $b_{s}(z)$ are defined close to those provided by experimental setup. In numerical calculations we used functions

$U_{s}=U_{0} /\left(1+\exp \left\{\left(z-z_{0}\right) / d_{1}\right\}\right)$,

$b_{s}=b_{0} /\left(1+\exp \left\{\left(z-z_{0}\right) / d_{1}\right\}\right)$,

for $-H<z<0$, as initial conditions with values $d_{1}$ and $z_{0}$ being parameters defining steepness of the profiles and initial stream locations. Values $U_{0}$ and $b_{0}$ correspond to characteristic stream velocity and turbulence values in experiment. The turbulent stream is assumed to be located in the region $z_{0}<z<0$, where both turbulence and mean velocity profiles remain unchanged during numerical calculations (i.e. maintained by the jet inductor) in the form of Eqs. (5b) and (5c).

Therefore, boundary conditions for the density fields at the top $(z=0)$ read

$\frac{\partial \rho}{\partial z}=0, \quad P=0, \quad$ for $z=0$.

Conditions at the bottom are imposed as

$\frac{\partial \rho}{\partial z}=0, \quad P=0, \quad U=0 \quad$ for $z=-H$.

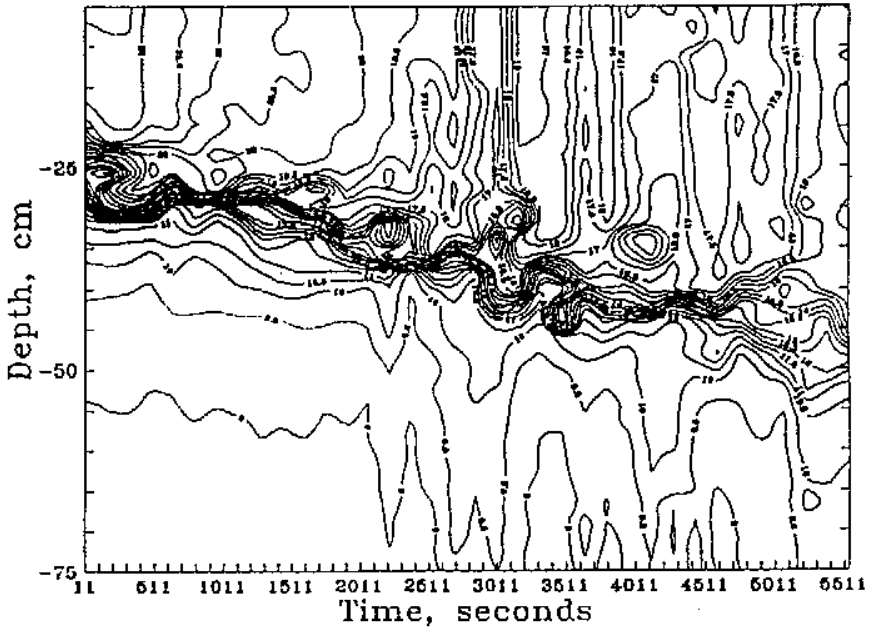

Fig. 5. Contour map of isotherms in experiment with $5 \mathrm{~cm} / \mathrm{s}$ mean flow velocity,

Initial density profiles were chosen close to observed in experiment in the form

$\rho_{s}=\rho_{0}+\Delta \rho_{0} / 2\left(1+\tanh \left\{\left(z-z_{01}\right) / d_{2}\right\}\right)$,

where $d_{2}$ and $z_{01}$ are parameters defining initial steepness of the density profile and thermocline location. Also, for the density fluctuations at $\mathrm{t}=0$ we have the condition

$P(z, 0)=0$.

We studied the problem of Eqs. (3-5) numerically using standard implicit code (Fletcher, 1990). Calculations were performed for three different values of free stream mean and turbulent (RMS) velocity values $U_{0}$ and $\left|u^{\prime}\right|=\sqrt{2 b}$. In the first run we used values $U_{0}=5 \mathrm{~cm} / \mathrm{s},\left|u^{\prime}\right|=3.5 \mathrm{~cm} / \mathrm{s}$, in the second run, $U_{0}=3 \mathrm{~cm} / \mathrm{s},\left|u^{\prime}\right|=1.7 \mathrm{~cm} / \mathrm{s}$, and in the third run $U_{0}=5 \mathrm{~cm} / \mathrm{s}$ and $\left|u^{\prime}\right|=1.5 \mathrm{~cm} / \mathrm{s}$ with all other parameters being fixed $\left(d_{1}=2 \mathrm{~cm}, d_{2}=4 \mathrm{~cm}, z_{0}=-16 \mathrm{~cm}\right.$, $z_{01}=-14 \mathrm{~cm}, T_{0}=4^{\circ} \mathrm{C}, H=70 \mathrm{~cm}$ ). Calculations were performed up to time $2000 \mathrm{~s}$ in the first and second runs and up to $3000 \mathrm{~s}$ in the 3rd run. All values of parameters used approximately correspond to those in the experiment, which enable us to perform some qualitative (and sometimes quantitative) comparison. However, the value of turbulent velocity fluctuations $\left(\left|u^{\prime}\right|=1.5 \mathrm{~cm} / \mathrm{s}\right)$ used in the third run is somewhat closer to the value observed in experiment than that in the first run $\left(\left|u^{\prime}\right|=3.5 \mathrm{~cm} / \mathrm{s}\right)$.

Results of numeric calculations are presented in Figs. 7, 8, 10 and 11.

Mean velocity and temperature fields, Brunt-Vaisala frequency and turbulent kinetic energy corresponding to various time moments obtained in the third run are shown in Fig. 10. Quantitatively, these are close to the profiles observed in experiment (cf. Figs. 2 and 3). Calculating dependencies of 


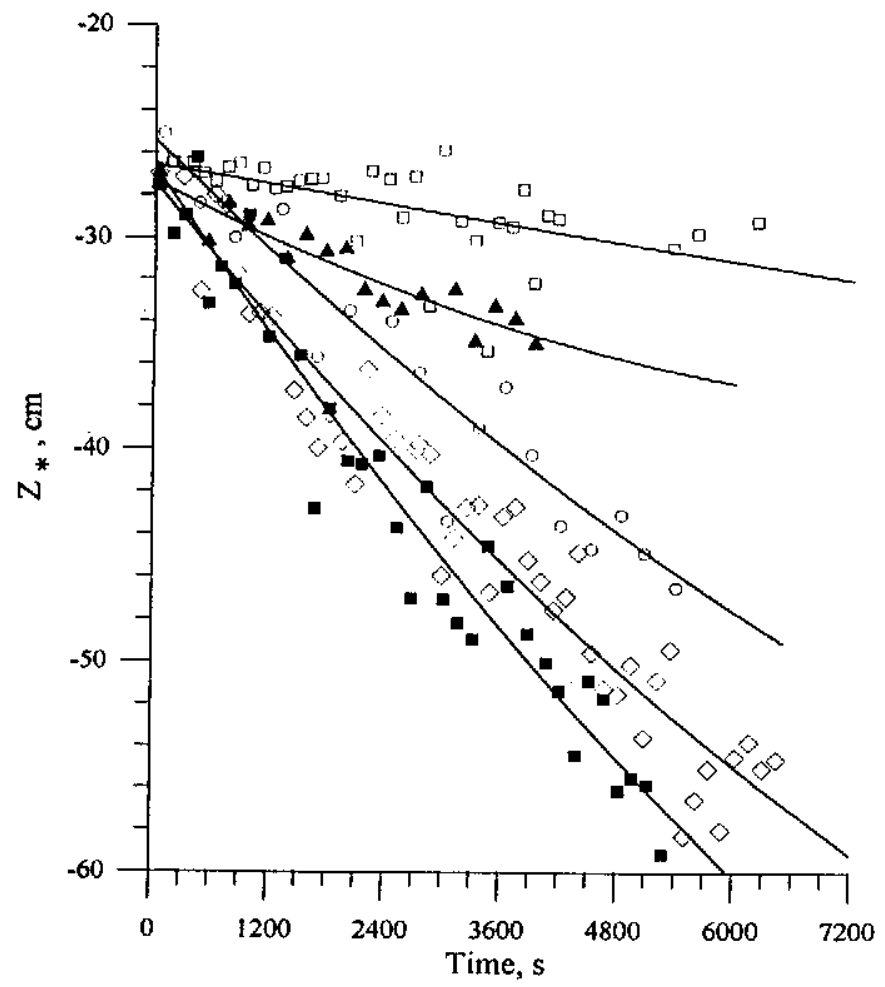

Fig. 6. Thermocline deepening: symbols represent the thermocline location: empty squares- $\langle U\rangle=2 \mathrm{~cm} / \mathrm{s}$, triangles- $\langle U\rangle=4 \mathrm{~cm} / \mathrm{s}$, circles- $\langle U\rangle=$ $5 \mathrm{~cm} / \mathrm{s}$, honeycombs- $\langle U\rangle=6 \mathrm{~cm} / \mathrm{s}$ and filled squares $-\langle U\rangle=7 \mathrm{~cm} / \mathrm{s}$.

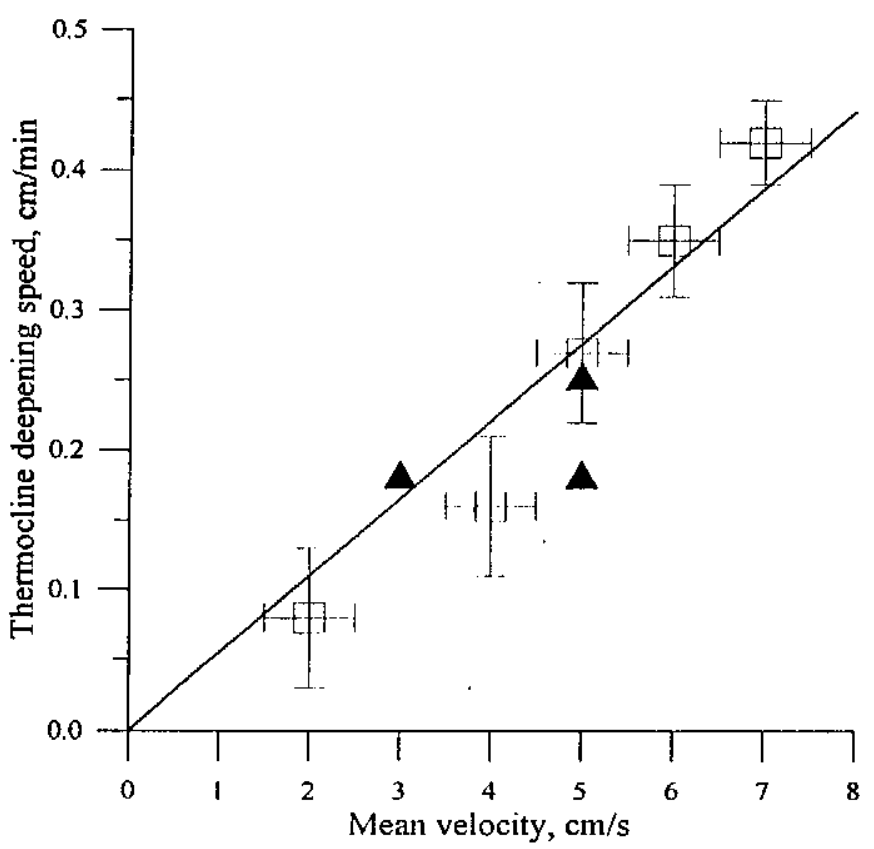

Fig. 7. Thermocline deepening rate. Squares with error bars-experiment, triangles-numerical data.

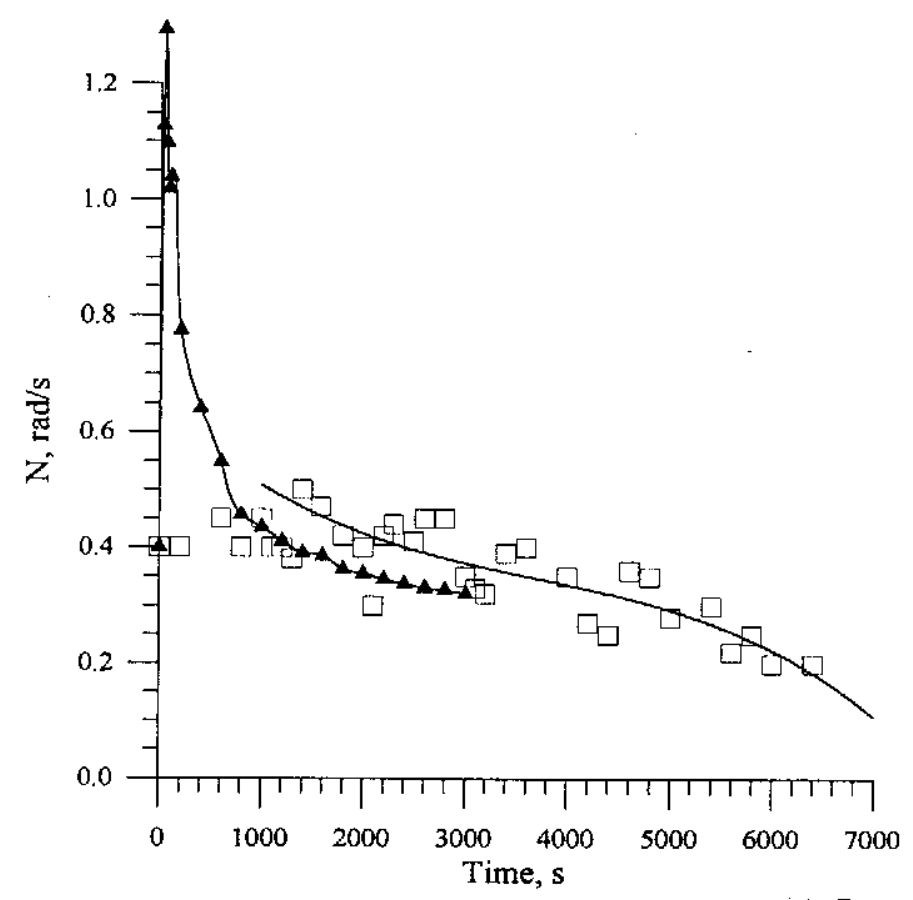

Fig. 8. Maximum of Brunt-Vaisala frequency vs time. Squares- experiment with $\langle U\rangle=5 \mathrm{~cm} / \mathrm{s}$, triangles- numerical computations for $\langle U\rangle=5 \mathrm{~cm} / \mathrm{s}$ and $\left|u^{\prime}\right|=1.5 \mathrm{~cm} / \mathrm{s}$.

maximum $\mathrm{N}$ and its location on time we obtained a good quantitative agreement with the experiment. Indeed, values of the deepening speed $(0.35 \mathrm{~cm} / \mathrm{min}$ and $0.18 \mathrm{~cm} / \mathrm{min}$ in the first and second runs, respectively) are close to those obtained in the experiment (Fig. 7). Numerical calculations give also quite accurate values for the maximum of the buoyancy frequency and its location (Figs. 8 and 11). Note that the value of the thermocline deepening speed obtained in the first run $(0.35 \mathrm{~cm} / \mathrm{min})$ is closer to the experimental observations than that in the third run $(0.17 \mathrm{~cm} / \mathrm{min})$. However, dependence of the thermocline location (maximum of $N$ ) on time obtained in the third run (filled triangles in Fig. 11) is more close to the experimental values. In numerical calculation we also obtained results describing early stages of thermocline dynamics (at times up to $200 \mathrm{~s}$ ) which indicate that there is an initial transient process accompanied by a sharp increase of mean temperature profile steepness and corresponding jump of $\mathrm{N}$. During this transient stage deepening speed reaches its maximum value (equal to $1.5 \mathrm{~cm} / \mathrm{min}$ and $0.96 \mathrm{~cm} / \mathrm{min}$ in first and second runs, respectively). As a result, quasi-stationary profiles of $\mathrm{U}, \mathrm{T}, \mathrm{N}$ and $\mathrm{b}$ are formed which change much more slowly at subsequent times. One can assume that this relatively fast transient corresponds to a bounce of the turbulent stream at the thermocline. 


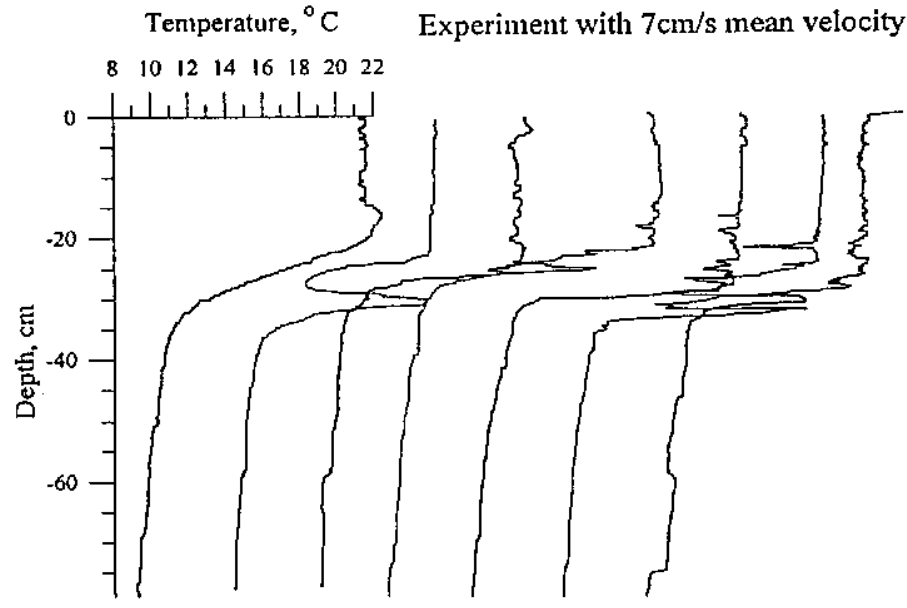

Fig. 9. Sequental temperature profiles measured with $1.5 \mathrm{~min}$ interval in experiment with $7 \mathrm{~cm} / \mathrm{s}$ mean flow velocity. The density inverse layers form at the beginning of experiment, resulting in "bursts" of microstructure.

The solution of Eq. (3d) showed a bright peak of temperature pulsations near the mixing interface. Since many experiments in stratified fluid are not provided with reliable velocity measurements, the profile of temperature fluctuations may be very informative.

Both experimental observations and numerical calculations show that after the short transient stage a quasi-stationary state of the thermocline is reached. This state corresponds to the situation, when the thermocline is located sufficiently below the stream region and deepens gradually at an approximately constant speed. In the upper well-mixed layer (above the thermocline location) gradients of the mean velocity and density fields are small and turbulent kinetic energy is substantial. Thus, one can neglect terms proportional to $(d U / d z)^{2}$ and $N^{2}$ in eq.(3a), so that corresponding equation for $b$ takes the approximate form

$\frac{\partial b}{\partial t}=\frac{\partial}{\partial z}\left(l \sqrt{b} \frac{\partial b}{\partial z}\right)-\frac{\gamma b^{3 / 2}}{l}$

with boundary condition

$b=b_{s}\left(z_{0}\right)$

at the edge of the stream.

The stationary solution of Eq. (6) can be written in the form

$$
\begin{aligned}
b(z) & =b_{s}\left(z_{0}\right) \exp \alpha\left(z-z_{0}\right), \quad z<z_{0}<0 \\
\alpha & =\sqrt{2 \gamma / 3 l} .
\end{aligned}
$$

The solution of Eq. (8) corresponds to exponential decay of turbulence with depth.

According to Eq. (8), for $|z| \ll 1 / \alpha$ turbulence energy is small as compared to the stationary value of $b$. Thus, if the thermocline, where mean field gradients $\partial U / \partial z$ and $\partial \rho / \partial z$ are large, is located sufficiently far from the stream, terms proportional to $b$ are comparatively small. Then, omitting terms proportional to $b$ in the Eq. (3a), we obtain the approximate balance condition in the form

$\kappa N^{2}=(\partial U / \partial z)^{2}$.

As seen from Eq. (9), far below the stream, in the thermocline region, the local Richardson number $R i=N^{2} /(\partial U / \partial z)^{2}$ should be close to the critical value defining the stability threshold (Monin and Yaglom, 1992):

$R i \simeq 1 / \kappa$.

Thus, even small shear disturbances (which may be caused, for example, by long internal waves) may be sufficient for the turbulence generation.

Numeric results are in good agreement with solutions (8) and (10). Profiles presented in Fig. 10 for the first run evidence that at later stages local Richardson number is close to value defined by Eq. (10) in the region of the thermocline and substantially exceeds this value in the other regions. In the experiment it was impossible to determine the local Richardson number with sufficient accuracy due to technical reasons (large experimental errors in velocity measurements). However, experimental data show relatively good agreement with numerical results. Namely, in the region of the thermocline characteristic values of the Richardson number are much smaller than in other regions.

\section{Conclusions}

In this paper some results of laboratory experiment are given dealing with the thermocline evolution forced by a turbulent stream in the upper layer, performed in large tank with thermal stratification. The results show that the thermocline inhibits a fast broadening of a jet in vertical plane and slowly deepens itself while remaining sharp enough. At the same time, a region of small Richardson number is formed which may stimulate the mixing process. A simple one-dimensional theoretical model based on $\mathrm{K}-1$ closure procedure is used to simulate the process numerically. It is remarkable that for comparatively low Reynolds numbers of the flow (few thousands and less) such a semi-empirical description gives a fairly good qualitative description of experimental results.

It is evident that both experimental and theoretical environment of this phenomenon can be (and should be) studied in more detail. For instance, the main weak point of this experiment is that a 3-dimensional flow pattern actually exists, which effects the dynamics of the whole system. The flow below the thermocline and close to the bottom of the tank was estimated using a hot wire anemometer and colorant tracers, and the velocities about $0.3-0.8 \mathrm{~cm} / \mathrm{s}$ were observed. These values are considerably less that those in the main flow region, but can complicate the matching of theoretical computations and experimental data. Moreover, more precise measurements for turbulence are desirable. Such processes as the fine structure formation and internal wave generation in experiment, and two- and three-dimensional description 

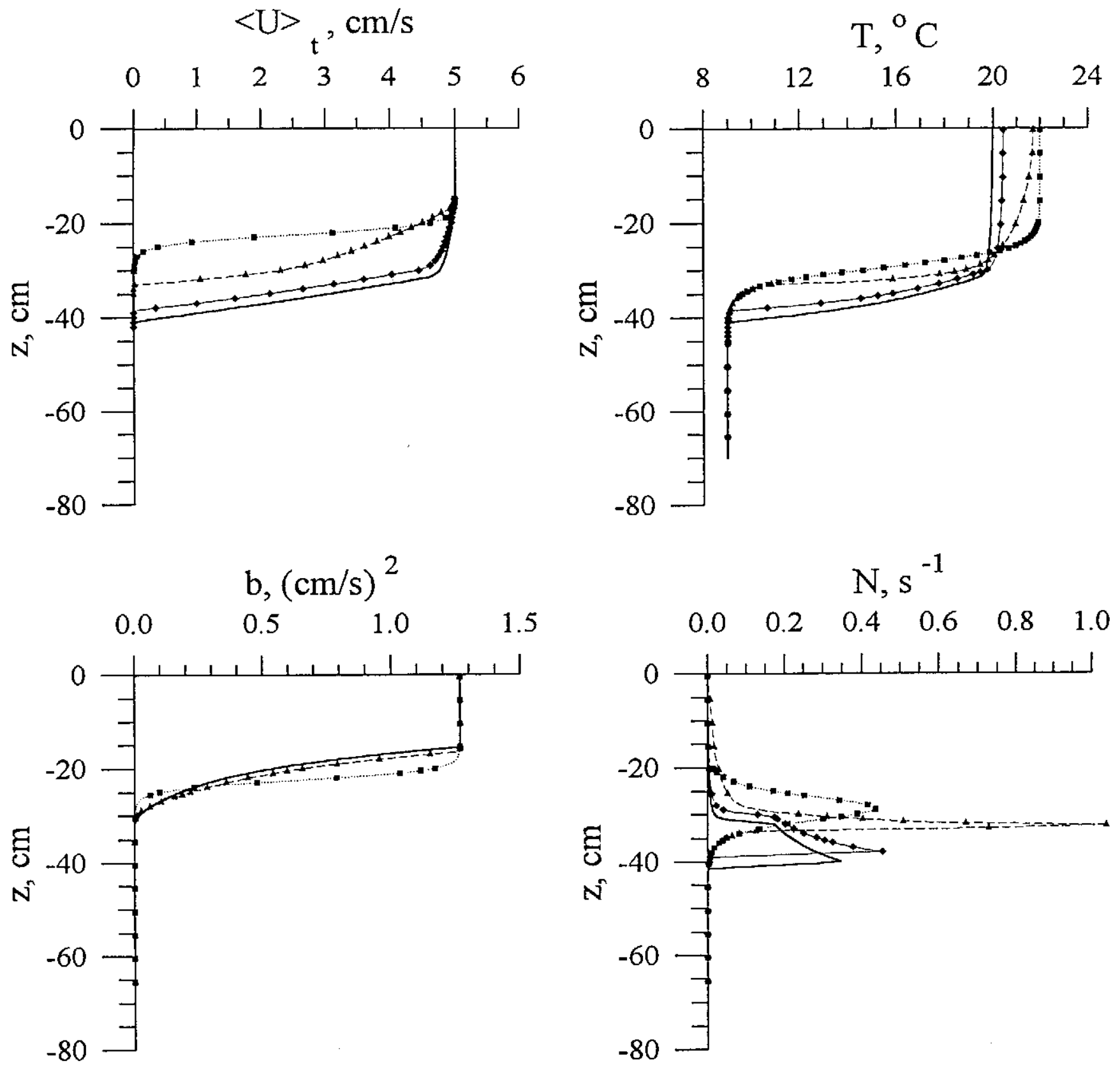

Fig. 10. Vertical profiles of mean velocity, temperature, turbulent energy and Brunt-Vaisala frequency obtained in numerical experiments for $\langle U\rangle=5 \mathrm{~cm} / \mathrm{s}$ and $\left|u^{\prime}\right|=1.5 \mathrm{~cm} / \mathrm{s}$. Squares and dotted curves- $t=0$, triangles and dashed curves- $t=100 \mathrm{~s}$, honeycombs and thin solid curves- $t=1000 \mathrm{~s}$ and thick solid curves $-\mathrm{t}=2000 \mathrm{~s}$. A profile of turbulent energy corresponding to $t=1000 \mathrm{~s}$ is very close to that for $\mathrm{t}=2000 \mathrm{~s}$ and not shown. 


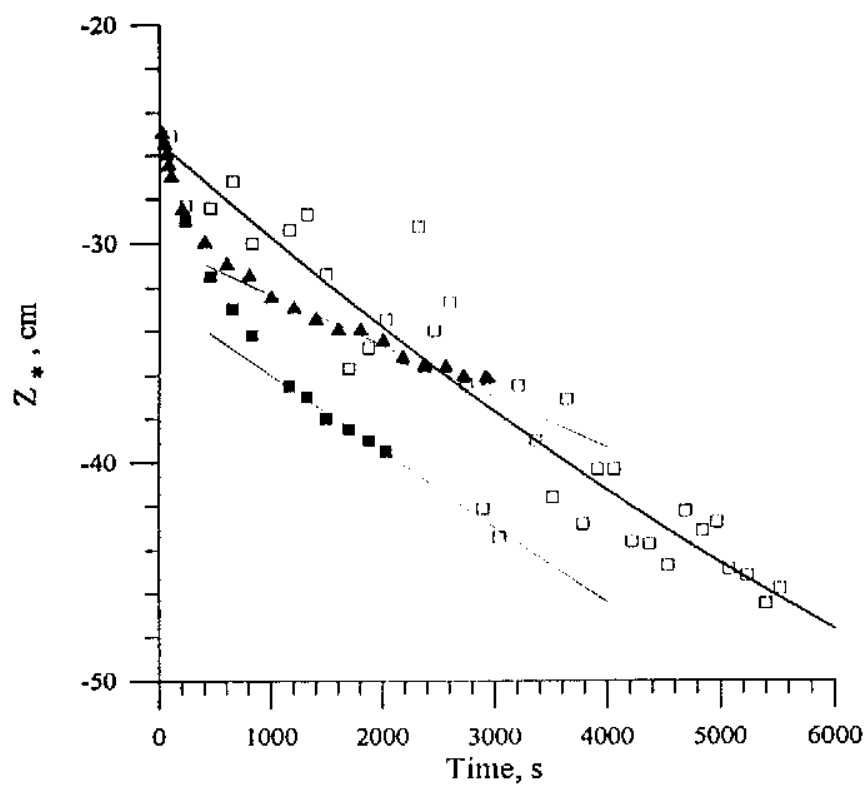

Fig. 11. Thermocline location: comparison of experimental and numerical data. Empty squares-experiment, triangles-numerical computations for $\langle U\rangle=5 \mathrm{~cm} / \mathrm{s}$ and $\left|u^{\prime}\right|=1.5 \mathrm{~cm} / \mathrm{s}$., filled squares-numerical computations for $\langle U\rangle=5 \mathrm{~cm} / \mathrm{s}$ and $\left|u^{\prime}\right|=3.5 \mathrm{~cm} / \mathrm{s}$.

are worthwhile to be specially investigated. At the same time the above results (seemingly the first ones as regards the submerged flow interaction with the thermocline) seem to be of use for understanding real geophysical processes and, in some aspects, even give the direct scaling of these processes.

Acknowledgements. The authors are very thankful to Dr. S. D. Bogatyrev and Prof. V. I. Talanov for the help and fruitful discussions.

\section{References}

Antonia, R. A., Chambers, A. J., Britz, D., and Browne, L., Organized structures in a turbulent plane jet: topology and contribution to momentum and heat transport, J. Fluid Mech, 172, 211, 1986.

Arabadzhi, V. V., Baranov, S. I., Bogatyrev, S. D., Brailovskaya, V. A., Kazakov, V. I., Kogan, V. R., Stepanyants, Y. A., and Talanov, V. I., Laboratory basin for the modeling of wave motions in the stratified ocean, in The Methods of Hydrophysical Research, edited by A. V. GaponovGrekhov and S. A. Khristianovich, Nizhny Novgorod, 1984.
Artale, V., Provenzale, A., and Santoleri, R. A., Process of thermocline erosion on the sicilian continental shelf, Nuovo Cimento, $11 C(5-6), 653-$ $665,1988$.

Britter, R, E., Laboratory experiments on turbulence in density-stratified fluids, Invited paper presented at the Eight Symposium of Turbulence and Diffusion, 1988.

Fletcher, C. A. J., Computational Techniques in Fluid Dynamics, vol. 1, Springer Verlag, 1990.

Gibson, C., Are Kelvin-Helmholtz billows inviscid or turbulent?, EOS, Trans. American Geophysical Union, 75(3, Supplement), 119, (abstract of report at the Ocean Sciences Meeting, San Diego), 1994.

Kato, H. and Phillips, O. M., On the penetration of a turbulent layer into stratified fluid, J. Fluid Mech., 37(4), 753-768, 1969.

Kazakov, V. I., Matusov, P. A., and Zaborskikh, D. V., Dynamics of microstructure in developing thermocline, in Report at the Ocean Sciences Meeting, San Diego, 1994.

Kennedy, P. J. and Shapiro, M. A., Further encounters with clear air turbulence in research aircraft, Journal of the Atmospheric Sciences, 37(5), 986-993, 1980.

Kreiman, K. D. and Bogdanov, V. E., Laboratory modelling of wind-induced entrainment in a stable stratified fluid, Izvestiya Akademii Nauk SSSR, Fiz. Atmi Okeana, 26(10), 1111-1119, 1990.

Monin, A. S. and Yaglom, A. M., Statistical Fluid Mechanics, 1, (in Russian) Moscow, 1992.

Ostrovsky, L. A. and Troitskaya, Y. I., A model of turbulence trasfer and dynamics of turbulence in a stratified shear layer flow, Izvestiya Akademii Nauk SSSR, Fiz. Atm.i Okeana, 23(10), (in Russian), 1987.

Perera, M. J. A. M., Fernando, H. J. S., and Boyer, D. L., Turbulent mixing at an inversion layer, J. Fluid Mech., 267, 275-298, 1994.

Rodi, W., Models of turbulence in surrounding media, in Prediction Methods for Turbulent Flows, Hemisphere, 1980.

Rodi, W., Examples of calculation methods for flow and mixing in stratified fluids, J. Geophys. Res., 92(C5), 5305-5328, 1987.

Sutherland, B. R. and Peltier, W. R., Turbulence transition and internal wave generation in density stratified jet, Phys. Fluids, 6, 1267-1284, 1994.

Xuequan, E. and Hopfinger, E. J., On mixing across an interface in stably stratified fluid., J. Fluid Mech., 166, 227-244, 1986.

Zilitinkevitch, S. S. and Mironov, D. V., A theoretical model of thermocline evolution in a freshwater basin, Izvestiya Akademii Nauk SSSR, Fiz. Atm.i Okeana, 25(9), 969-978, 1989.

Zilitinkevitch, S. S., Kreiman, K. D., and Felzenbaum, A. I., Turbulence, heat exchange and self-similarity of temperature profile in thermocline, Doklady Akademii Nauk SSSR, 300(5), 1226-1230, 1988. 